\title{
La construcción del ocio en adolescentes y su influencia en el desarrollo de trayectorias delictivas
}

\author{
The leisure construction in adolescents and this influence \\ for development of criminal careers
}

\author{
José Javier NAVARRo PÉREZ \\ Universitat de València \\ jjnavper@uv.es \\ Francesc Xavier UCEDA I MAZA \\ Universitat de València \\ franuma@uv.es \\ José Vicente PÉREz Cosín \\ Universitat de València \\ jvperez@uv.es
}

Recibido: $23 / 01 / 2003$

Revisado: 20/05/2003

Aceptado: 15/07/2013

Disponible on line: 20/12/2013

\section{Resumen}

La construcción de actividades de ocio incide directamente en el desarrollo evolutivo de los adolescentes, capacitándolos para una sana socialización en sus espacios cotidianos. La complejidad que plantea el diseño de actividades y programas de ocio requiere el esfuerzo común de los diferentes agentes; así, tanto las administraciones públicas, como los padres, profesionales, los propios adolescentes y las entidades gestoras de estos programas han de coordinar sus esfuerzos en pro de desarrollar equilibradamente los espacios con las propuestas para el ocio. De estas actividades, las encaminadas hacia el recreo supervisado permiten crecer en positivo, pero la alarma aparece cuando los espacios para el ocio, se convierten espontáneamente en guarida para el desarrollo de actividades opacas, transformando espacios dedicados a la diversión en prácticas de riesgo. Llegados a este punto, el presente artículo demuestra que la forma de disfrutar del ocio influye en el desarrollo de actividades delictivas y que a mayor ocio desestructurado, mayor profundización en la trayectoria delictiva. Para ello se sigue una estrategia de complementariedad metodológica, cuyos datos cuantitativos se extraen de los expedientes de 281 adolescentes en conflicto con la ley de la ciudad de Valencia y se vinculan a tres trayectorias delictivas generadas a partir del análisis multivariable. Asimismo se completa con entrevistas a informantes claves que participan en los escenarios de los adolescentes en conflicto con la ley, desde el plano judicial, educativo, policial o de la intervención social. Se concluye que el ocio constituye un escenario ideal para el empoderamiento de los adolescentes ${ }^{1}$.

Palabras clave: Adolescentes, riesgos, ocio, participación, trayectoria delictiva.

\begin{abstract}
The construction of leisure activities affects directly in the positive development of the teens, enable them to a healthy socialization of their lives. The complexity of the activity design and leisure programs requires a big effort by the different agents; so the public administrations, parents, professionals, teens and the management of these programs have to coordinate their efforts to develop a balanced space for the leisure activities. The activities that focus on the supervised leisure allow to grow positively. The alarm arises when the leisure spaces change without any notice into dangerous risk spaces. In this sense, this article will attempt to explain how the teen build their behaviours, leisure situations and how they are capable of generate incorrect dynamics that contribute in the negative development of the teen. Furthermore, this article will try to discover how to define public practices and to see how youngsters build their participation strategies and educational promotion to play constructive playgrounds. All is done with the intention to provide with the right information to successful professionals to be able to diagnose effectively. At this point, this article
\end{abstract}

${ }^{1}$ El presente artículo se proyecta en el marco del proyecto de investigación que lleva por título «El sistema de Servicios Sociales ante la crisis económica. Retos, desafíos y territorio: el caso de la provincia de Valencia» Ref./ UV-INV-PRECOMP12-82190. 
shows how to enjoy leisure influences the development of crime. A more leisure deviated risk of incurring more crimes. This strategy is developed methodological complementarity, where quantitative data records are extracted from 281 adolescents in conflict with the law of the city of Valencia and linked to three criminal trajectories generated from the multivariate analysis. The article is completed with privileged informant interviews everyday spaces shared with adolescents in conflict with the law, since the legal field, educational, police or social intervention. Among the most important findings, we found that leisure is important rehabilitative tool for empowerment of adolescents.

Keywords: Teens, risks, leisure, participation, criminal career.

Referencia normalizada: Navarro Pérez, J. J., Uceda i Maza, F. X., y Pérez Cosín, J. V. (2013): «La construcción del ocio en adolescentes y su influencia en el desarrollo de trayectorias delictivas». Cuadernos de Trabajo Social, 26(2): 455-465.

Sumario: 1. Las adolescencias y el ocio: desviación y prácticas de riesgo. 2. El diseño metodológico de la investigación. 3. Los resultados y su análisis. 4. Conclusiones y propuestas. 5. Referencias bibliográficas.

\section{Las adolescencias y el ocio: desviación y prácticas de riesgo.}

En nuestra cultura occidental, la adolescencia como etapa evolutiva cada vez adquiere más importancia, por ello se convierte en objeto de estudio de la biología, medicina, psicología, sociología, antropología, trabajo social, etc. (Bueno Abad y Pérez Cosín, 1998). Si bien se considera una etapa evolutiva, la condición adolescente es algo más que un grupo de edades o unas características evolutivas, por lo que es necesario visiones poliédricas (Funes, 2005).

En nuestro entorno, estamos en las primeras generaciones de adolescentes postmodernos, donde el ocio juega un papel fundamental en la socialización (Tarín y Navarro, 2006). El ocio en sí mismo, es capaz de prevenir y de promocionar simultáneamente. Hablamos de ocio constructivo cuando el escenario lúdico converge con espacios para el aprendizaje, favoreciendo la configuración de competencias que incitan a la reflexión en los adolescentes.

Los factores relacionados con la participación y la presencia activa de los adolescentes en actividades de ocio, deberán ser el marco de referencia para la articulación de prácticas educativas en los espacios de socialización que a su vez favorezcan el intercambio, la conexión hacia nuevos y atractivos retos tratando de lograr además, que los espacios lúdicos sirvan de instrumento para la inclusión social.

En este sentido Fernández Barrera (2009) señala que «la participación tiene que ver con el ejercicio de la palabra y la posibilidad de los niños y niñas de actuar en todas aquellas áreas de su vida cotidiana que son de su interés» (p. 115). Desde la primera infancia, los niños se inician en el entrenamiento de los afectos en combinación con los elementos propios de lo lúdico; ambas dimensiones potenciarán otras esferas que conformarán la personalidad del adolescente (Úcar, 2001). Razón principal por la que entendemos prioritaria la gestión constructivo-educativa de los espacios de ocio, articulando para ello nuevas y atractivas vías para la inclusión de los y las adolescentes.

En la sociedad postmoderna, la vulnerabilidad y el riesgo de exclusión alcanzan a sectores más amplios de la sociedad; los riesgos se han ampliado y diversificado haciéndonos más vulnerables en extensión e intensidad. Castel (1997) señala que la sociedad actual se caracteriza por la consolidación de una extensa zona de vulnerabilidad antesala de la exclusión. El reto es comprender cómo la creciente vulnerabilidad y exclusión social afecta a los adolescentes y a su proyección de futuro. En este sentido, los factores de vulnerabilidad están más relacionados con las actividades vinculadas a las causas que generan el riesgo en los adolescentes (carecer de actividades inclusivas durante los procesos absentistas o de ausencia escolar), que con el riesgo o actividad desviada en sí misma (absentismo escolar o no acudir a la escuela). Así, el tiempo de exposición al riesgo configurará el grado de vulnerabilidad al que se somete el adolescente, sobre el que la precariedad y las fragilidades desgastarán los resortes de la actividad productiva. 
La acción de la justicia penal de menores ${ }^{2}$ se articula sobre las conductas desviadas de los adolescentes, configurando pues un importante termómetro de la situación social que atraviesan y de las políticas que tratan de prevenir y controlar estas acciones que truncan el desarrollo ajustado de los adolescentes. En este sentido señala Navarro (2009) que el crecimiento en las tasas de desviación de la juventud, configura uno de los más graves indicadores de insuficiencia de las estructuras y niveles de control que representan a la sociedad civil.

El uso del tiempo de ocio, es fundamental para la socialización de los adolescentes, ya se encuentren en conflicto con la ley o no. Asimismo es un espacio educativo, preventivo y generador de identidad, y en nuestra sociedad podemos afirmar que de ciudadanía (Savater, 1997; Pérez Cosín y Uceda, 2009). El ocio como construcción social, es fundamental en la socialización y en el binomio integración-vulnerabilidad forma parte de los derechos humanos individuales y colectivos, y concebido como una práctica individual y colectiva articulada a modo de capital social que se inscribe en las biografías de los sujetos y en sus vidas en común.

En este contexto, como ha señalado Cuenca (2004, p.72), el disfrute y el ejercicio del ocio, sea a nivel personal o comunitario, no depende tan sólo del hecho de disponer de tiempo y de recursos sino, «y de un modo especial, de la percepción y actitud que se tiene sobre el mismo»; así pues, la gestión del ocio por los propios adolescentes a través de redes estables de relación, constituye un proceso que pondera más si cabe la diversión y el desarrollo madurativo en clave positiva. En este sentido Lévesque y Robert (2008), plantean que los adolescentes que planifican su tiempo libre a través de grupos no consolidados, asumen un riesgo adicional atendiendo a los diferentes niveles y entornos requeridos para cimentar la relación.

Por el contrario, en espacios seguros, comprometidos y autogobernados por ellos, los propios adolescentes aprenden a ganar, a perder, a compartir, a implicarse, a asumir tareas individuales y de grupo, en una palabra, los espacios de ocio que han sido creados por grupos consistentes de adolescentes permiten la auto- gestión de sus dinámicas y que después estas puedan verse reflejadas en otras esferas; los lazos de amistad, la reciprocidad, la lealtad a los acuerdos, la articulación de normas constituyen interesantes respuestas que el marco del ocio permite entrenar.

Las «buenas prácticas» (ocio activo, constructivo, creativo, lúdico, participativo, experiencial, etc.), todavía van de la mano de los «contratiempos» que también el ocio evoca en nuestras sociedades, confundiéndolo con ociosidad, banalidad, consumismo, vagancia, pere$\mathrm{za}$, indolencia, etc. Mantener el anclaje en estas últimas, cuando - como explica Lasén (2000, p.170) - el «ocio adquiere un valor tácito en la conquista del saber y en el sentido dado a la existencia», permite la creación y recreación de nuevos vínculos sociales, gracias a la densidad e intensidad humana que está ausente en otras situaciones, ya no es ignorancia o desdén, sino simple ceguera a lo que el mundo y sus tiempos están cambiando.

Debemos hacernos eco del modelo de ocio característico de las sociedades postindustriales. Una oferta apetitosa para disfrutar en familia (cines, centros comerciales, espectáculos, etc.), pero que deja a los adolescentes descolgados. Si echamos un vistazo a las actividades, han de pasar por la esfera del deporte o por los movimientos diocesanos/escoltas. Difícilmente podemos encontrar alternativas de ocio medioambientales, que inciten a la representatividad juvenil, vinculadas a la solidaridad, en definitiva que desarrollen los afectos, los valores, que potencien la reflexión, etc. Atendiendo a los planteamientos de Del Barrio (2002) «los valores no pueden afianzarse con procedimientos meramente declarativos»(p. 304). Ello supone que es importante que el ocio se oriente y se encauce desde instancias que abiertamente manifiesten una opción lúdico-constructiva positiva.

También debemos recordar las «malas prácticas» que los adolescentes vinculan a los tiempos de ocio (Caballo, Caride y Meira, 2011). El problema de convertir el ocio en una rutina, supone que todo el tiempo, se convierte en tiempo libre, en que lo anodino se hace habitual y donde los espacios de ocio quedan subyugados a las inercias del tiempo. Esto supone iniciar la espiral de riesgo a través del mismo ocio, lo que

\footnotetext{
${ }^{2}$ En el caso de España, tipificadas penalmente a través de la LORPM 5/2000.
} 
nos lleva a la conclusión de que esta banalización del ocio o infrautilización del tiempo libre, viene determinado básicamente por cuatro elementos: 1) Las situaciones marcadas por la ausencia de intercambio relacional (siempre los mismos, en el mismo sitio y haciendo las mismas cosas); 2) La disminución de los estímulos positivos y motivadores ${ }^{3}$ (contacto limitado a lo inmediato, pasivo e inamovible); 3) Rechazo al ambiente educativo, ya que por norma general este incita a la movilización y 4) Ausencia de identificación con ambientes normalizados sobre los que poder articular dinámicas de relación positiva.

Podríamos señalar que el ocio puede jugar un factor de protección frente al desarrollo de conductas desviadas como la delincuencia juvenil o representar en sí mismo un componente aditivo, un factor de riesgo más, atendiendo la investigación de Catalano y Hawkins (1996) en la que señalan que las conductas desviadas se producen por la abundancia de factores de riesgo sin que existan factores de protección que ejerzan un influjo de compensación.

\section{El diseño metodológico de la investigación}

Se aborda la investigación desde la pluralidad metodológica, combinando e integrando diferentes perspectivas, técnicas y enfoques que, lejos de ser excluyentes, adquieren un carácter de complementariedad. Los resultados aquí presentados forman parte de una investigación más amplia donde el objetivo principal es identificar las vulnerabilidades sociales y los procesos de exclusión de los adolescentes en conflicto con la ley, para establecer la relación entre la vulnerabilidad y la construcción de las trayectorias delictivas y diseñar programas de prevención en los espacios comunitarios (Uceda, 2012). Por ello uno de los objetivos específicos es explicar el papel del ocio como variable de vulnerabilidad en las trayectorias delictivas, al que responde este artículo. En este sentido las hipótesis que guían esta sección de la investigación son: 1. La forma de vivir el ocio influye en la adopción de conductas delic- tivas por los adolescentes; 2 . A mayor ocio desestructurado, mayor profundización en la trayectoria delictiva y 3 . El ocio es un espacio privilegiado para el diseño de estrategias de prevención primaria y secundaria.

Se inició el trabajo a partir del análisis de fuentes primarias, es decir consultando individualmente los expedientes de los Adolescentes en conflicto con la ley de la ciudad de València en el año 2006. Fueron un total de 286 los consultados (aunque sólo había datos válidos en 281 expedientes que fueron los utilizados en la investigación). De los informes sociales que acompaña cada medida judicial se extrajo la variable Ocio, tal y como la registraban los trabajadores sociales del equipo técnico: a) Ocio desestructurado o «callejeo»; b) Ocio programado; c) Ocio solitario y d) No consta (es decir no constaba al expediente la forma de ocio de Adolescentes en conflicto con la ley. Preguntados los trabajadores sociales señalaban que en el informe social se registraba la variable «ocio», si se consideraba un factor que influyera tanto desde el punto de vista de la protección (ocio programado) como de la vulnerabilidad (ocio desestructurado o «callejeo»). En el caso de «no constar» se explica por su parte por qué no era considerado factor relevante en la situación de estos Adolescentes en conflicto con la ley.

Algunos datos socio demográficos de la muestra son: a) el 82 por ciento chicos, el 18 por ciento chicas; b) el 22 por ciento pertenece al pueblo gitano frente al 75 por ciento y en el 2 por ciento de los casos se desconocía; c) el 76 por ciento proceden de España y el 22 por ciento de procesos migratorios $(7$ por ciento del Magreb, 8 por ciento América Latina, 5 por ciento Europa del Este y 2 por ciento de países subsaharianos). No existe un análisis diferenciado en función de estos datos pues el criterio utilizado es su ubicación en una trayectoria delictiva u otra frente a la variable.

A partir de aquí, la estrategia que se siguió es observar la variable ocio como se comportaba en las tres trayectorias generadas a partir del SPSS.16 ${ }^{4}$ : a) Adolescentes en conflicto con la

\footnotetext{
${ }^{3}$ Inevitablemente la socialización y los modelos de referencia se verán condicionados en este sentido.

${ }^{4}$ El análisis estadístico multivariable se ha realizado a partir del estudio de los 281 expedientes, generando 20 variables complejas por modificación, suma, depuración o producto, con el resultado de 172 variables en el archivo. Este último procedimiento ha supuesto elaborar un índice complejo como resultado final o variable total que consideramos criterio integrador a nivel cuantitativo de las trayectorias de los Ado-
} 
ley de trayectoria delictiva inicial (ACLTI $)^{5} ; \mathrm{b}$ ) Adolescentes en conflicto con la ley de trayectoria delictiva moderada (ACLTM $)^{6}$, y c) Adolescentes en conflicto con la ley de trayectoria delictiva consolidada (ACLTC) ${ }^{7}$.

Una vez comprobada que la variable ocio es significativa en las trayectorias decidimos profundizar en su explicación mediante el trabajo cualitativo a partir de entrevistas en profundidad a informantes clave. Se ha seguido la propuesta de Patton (1990), en cuanto a la realización de las entrevistas apoyadas en un guión previo. Se han realizado 32 entrevistas a profesionales de la intervención social con Adolescentes en conflicto con la ley que han sido analizadas con el apoyo del programa informático MAXqda. Los informante claves fueron seleccionados del conjunto de escenarios que intervienen con los Adolescentes en conflicto con la ley, «siguiendo criterios de inclusión que respondieran a la heterogeneidad, accesibilidad y representación» de acuerdo con Vallés (1999, p. 89). Se estableció como criterio básico que hubieran desarrollado su actividad laboral con Adolescentes en conflicto con la ley durante más de cinco años (Tabla 1).

\section{Los resultados y su análisis}

Iniciamos el análisis con los datos cuantitativos y seguiremos con los datos cualitativos. En primer lugar, veremos la relación entre trayectoria delictiva y el ocio para comprobar similitudes y diferencias en cada una de las trayectorias predefinidas (Tabla 2).

En los ACLTI, el ocio fue calificado como desestructurado y de «callejeo» en el 47,9 por ciento, como «ocio programado» en el 11,7 por ciento, «ocio solitario» en el 5,3 por ciento, y no constaba en el 35,1 por ciento. En los ACLTM, el ocio fue calificado como desestructurado y de «callejeo» en el 72,1 por ciento, como «ocio programado» en el 3,1 por ciento, «ocio solitario» en el 4,1 por ciento, y no constaba en el 20,7 por ciento. En los ACLTC, el ocio fue calificado como desestructurado y de «callejeo» en el 92,2 por ciento, como «ocio programado» en el 2,2 por ciento, «ocio solitario» en el 2,2 por ciento, y no constaba en el 3,3 por ciento.

Como se observa es evidente que la variable ocio ocupa un espacio significativo, pues conforme el adolescente en conflicto con la ley se encuentra en una trayectoria delictiva más desarrollada disminuye de forma importante el «no consta» en los informes de los trabajadores sociales, asimismo en la medida que se profundiza en la trayectoria delictiva aumenta de forma importante el ocio desestructurado o «callejeo» y disminuye el ocio programado.

Veamos a continuación los discursos de los profesionales que intervienen con los adolescentes en conflicto con la ley y sus consideraciones al respecto:

En los adolescentes existe un ocio que se puede clasificar de «estar», es decir, estar con los colegas aparentemente sin una actividad estructurada, compartiendo momentos en el parque, en la calle, siendo una de las experiencias de pertenencia e identidad de grupo fundamental. Acercando el enfoque desde otro ángulo, ello incide directamente en la reducción de la creatividad que los adolescentes reproducen en sus espacios de ocio. Hay que tener en cuenta que les viene todo asociado a un sistema cerrado, como en un pack y que a veces imaginarse otros momentos y otros tiempos, implica ir más allá de los que les viene «envasado»:

Tú, fijate y verás que hay muy pocas cosas que hoy día puedan transformarse. Está todo muy estudiado para que no tengan que inventar nada. Es un copia y pega (EMAE-4).

La mayoría lo utilizan para estar con otros en parques, hablando o fumando. Sólo estar. Creo que ha cambiado respecto de antes porque

lescentes en conflicto con la ley, estableciendo para ello tres situaciones diferenciadas, es decir: ACLTI, ACLTM y ACLTC.

${ }^{5}$ Correspondiente al primer tercil, son 94 casos y su trayectoria delictiva se encuentra definida por haber cometido un delito, disponer de un tipo de medida y únicamente haber pasado por un juzgado.

${ }^{6}$ Correspondiente con el segundo tercil, son 97 casos y su trayectoria delictiva se ha concretado en haber pasado por 1,5 juzgados (es decir, tan habitual es haber pasado por uno como por dos) y haber cometido dos delitos pero el tipo de medida impuesta es una;

${ }^{7}$ Correspondiente con el tercer tercil, son 90 casos, han pasado por cuatro juzgados, han cometido cuatro delitos y el tipo de medida impuesta son de tres diferentes. 


\begin{tabular}{|c|c|c|}
\hline Ámbito & Perfil profesional & Codificación \\
\hline Escenario judicial: ámbito jurídico & $\begin{array}{l}\text { Abogado } \\
\text { Abogado } \\
\text { Abogado } \\
\text { Jueza } \\
\text { Juez } \\
\text { Juez } \\
\text { Fiscala } \\
\text { Fiscala } \\
\text { Fiscala }\end{array}$ & $\begin{array}{c}\text { Entrevista/EAB1 } \\
\text { Entrevista/EAB2 } \\
\text { Entrevista/EAB3 } \\
\text { Entrevista/EJ1 } \\
\text { Entrevista/EJ2 } \\
\text { Entrevista/EJ3 } \\
\text { Entrevista/EFIS1 } \\
\text { Entrevista/EFIS2 } \\
\text { Entrevista/EFIS3 }\end{array}$ \\
\hline $\begin{array}{l}\text { Escenario judicial: ámbito psico-social } \\
\text { (equipo técnico) }\end{array}$ & $\begin{array}{c}\text { Téc. Esp. Menor } \\
\text { (Pedagogo) } \\
\text { Téc. Esp. Menor } \\
\text { (Maestra) } \\
\text { Trabajadora social } \\
\text { Trabajadora social } \\
\text { Psicóloga } \\
\text { Psicóloga } \\
\text { Psicóloga }\end{array}$ & $\begin{array}{c}\text { Entrevista/EETTM1 } \\
\text { Entrevista/EETTM2 } \\
\text { Entrevista/EETTS1 } \\
\text { Entrevista/EETTS2 } \\
\text { Entrevista/EETP1 } \\
\text { Entrevista/EETP2 } \\
\text { Entrevista/EETP3 }\end{array}$ \\
\hline Escenario judicial ámbito psico-social medio abierto & $\begin{array}{l}\text { Educador social } \\
\text { Educador social } \\
\text { Educador social } \\
\text { Educador social }\end{array}$ & $\begin{array}{l}\text { Entrevista/EMAE1 } \\
\text { Entrevista/EMAE2 } \\
\text { Entrevista/EMAE3 } \\
\text { Entrevista/EMAE4 }\end{array}$ \\
\hline Escenario judicial ámbito psico-social medio cerrado & $\begin{array}{c}\text { Educador } \\
\text { Educadora } \\
\text { Educadora } \\
\text { Educador } \\
\text { Educador } \\
\text { Trabajador social } \\
\text { Psicóloga }\end{array}$ & $\begin{array}{l}\text { Entrevista/ECOLE1 } \\
\text { Entrevista/ECOLE2 } \\
\text { Entrevista/ECOLE3 } \\
\text { Entrevista/EDIAE1 } \\
\text { Entrevista/EDIAE2 } \\
\text { Entrevista/ECOLTS } \\
\text { Entrevista/ECOLP1 }\end{array}$ \\
\hline Escenario judicial-ámbito ejecución unidad docente & $\begin{array}{l}\text { Profesora } \\
\text { Profesora } \\
\text { Profesora }\end{array}$ & $\begin{array}{l}\text { Entrevista/EMEST1 } \\
\text { Entrevista/EMEST2 } \\
\text { Entrevista/EMEST3 }\end{array}$ \\
\hline Escenario policial & $\begin{array}{l}\text { Inspectora } \\
\text { Subinspector }\end{array}$ & $\begin{array}{l}\text { Entrevista/EGRUME } \\
\text { Entrevista/EPN }\end{array}$ \\
\hline
\end{tabular}

Tabla 1. Relación de informantes clave: ámbito, perfil profesional y codificación.

Fuente: Elaboración propia.

\begin{tabular}{|l|c|c|c|}
\hline & $\begin{array}{c}\text { ACLTI } \\
\text { (porcentaje) }\end{array}$ & $\begin{array}{c}\text { ACLTM } \\
\text { (porcentaje) }\end{array}$ & $\begin{array}{c}\text { ACLTC } \\
\text { (porcentaje) }\end{array}$ \\
\hline Ocio desestructurado y de «callejeo» & 47,9 & 72,1 & 92,2 \\
No consta & 35,1 & 20,7 & 3,3 \\
Ocio programado & 1,7 & 4,1 & 2,2 \\
Ocio solitario & 5,3 & 3,1 & 2,2 \\
\hline
\end{tabular}

Tabla 2. Variable de ocio y tiempo libre y relación con las trayectorias Fuente: Elaboración propia. 
antes las actividades del momento comprendían actividades deportivas en grupos; eso se ha acabado. Los fines de semana la cosa cambia, porque van a discotecas, $p u b$ 's, ahí se mezclan todos; los delincuentes y los no delincuentes (EETTM-1).

Por otro lado, hallamos un ocio individualizado, en el no hay intercambio ni comunicación interpersonal no se comparten emociones, sino que se queda atrapado por la esfera del espacio individual:

En general, tienen Playstation o van a casa de algún amigo que la tiene o al parque. No juegan al fútbol ni están apuntados a ninguna historia (EETP-2).

Existe un paso clave del estar ocioso a un ocio desestructurado o denominado por muchos de ellos de «callejeo». El primero se caracteriza por el estar, y el segundo es cruzar una línea difusa donde las horas sin hacer nada acaban siendo horas de riesgo, etc. En este sentido, podríamos identificar que las rutinas cotidianas se convierten en contextos de riesgo y ello eleva las posibilidades de vulnerabilidad. También existe una limitada supervisión familiar y posturas alejadas de la educación formal respecto de la recuperación de actividades de ocio estructuradas en los espacios formales. Unido a la lejanía de intereses entre adultos y adolescentes que acaban condicionando el desarrollo de acontecimientos desviados:

Hay menores que no tienen ningún control por parte de nadie y salen mucho, están en la calle, se acuestan a las 5, se levantan a la una y no pasa nada porque no tienen ninguna responsabilidad. Se fuman unos porros y es tan normal para ellos que lo hagan todos los días (EETTM-2).

El límite se lo pone cada nano, porque en su casa pues no le dicen, «oye tú que esto ahora no lo puedes hacer, que tal [...], los padres hacen la vista gorda y tema zanjado [...] luego modular todo esto ya se hace más cuesta arriba» (EETTS-2).

Las oportunidades de las que disponen los adolescentes de hoy y el tipo de ocio que la sociedad oferta para ellos es escasa; se deduce de los discursos, que se basa en un ocio muy consumista, en el aquí y ahora y en las capacidades económicas. Habría que realizar un inciso en este sentido, para explicar que las posibilidades han globalizado los espacios y los tiempos de ocio. Aquí no hablamos ya de discotecas para los que residen en entornos con recursos y otras para los precarizados, o centros comerciales dirigidos a uno $u$ otro tipo de consumo, sino que los lugares no tienden a generar etiquetas, porque estas han sido eliminadas por la propia socialización del consumo:

Es mimetismo puro, esperar que alguien vaya con coche e ir a la discoteca de turno, así todo el fin de semana [...] el consumismo influye en cuanto que todo lo que hacen cuesta dinero (ECOLE-1).

Piensa que en la discoteca todo distorsiona y ahí no se pregunta si tu vives en un chabolo o en un ático en el centro. Una vez estas dentro, pues a pasarlo bien, seas de donde seas. (EETPP-2).

El ocio en nuestra sociedad esta segmentado, es decir, existe un ocio y espacio totalmente diferenciado para los adolescentes, jóvenes $\mathrm{y}$ adultos siendo un aspecto clave en la socialización. Observamos un desconocimiento entre el ocio de los adultos y el de los adolescentes:

Antes la chavalería estaba y había una presencia adulta, con una transmisión de la experiencia adulta que siempre es saludable [...] ahora yo hago excursiones a centros de ocio y te das cuenta que por encima de 21 años no hay nadie, es decir, los camareros son jovencísimos, los policías nacionales tienen 21 años porque el turno de noche se queda exclusivamente para los jóvenes (EMAE-4).

¿Cuántos padres has visto tú con unos patines haciendo skate en una olla? Pues, como eso, todo» (EECOLTS).

Un factor de prevención primaria y de protección secundaria es la participación de los adolescentes en actividades de ocio estructuradas, ya sean deportivas, de tiempo libre, etc. Añadiremos, en sentido negativo, que la inconsistencia en las actividades de ocio, genera también el acceso a vías alternativas per sé, desviadas del ocio integrado:

Los que tienen un ocio estructurado pueden venir una, dos o tres veces, pero por cuestiones de grupo se dejan influir y se meten en líos, pero no suelen mantenerse en las conductas infractoras (EETTS-1). 
La complementariedad con los datos cualitativos nos aporta validez a la interpretación cuantitativa. Es decir, son una minoría los adolescentes que disfrutan de un ocio supervisado, estructurado - que no quiere decir vigiladoy conforme aumenta su trayectoria delictiva se produce un incremento de un ocio de riesgo, que ocupa la mayor parte de su cotidianeidad. Estos datos coinciden con la investigación de Torrente y Rodríguez (2004), confirman el hecho de que a mayor contacto con actividades cotidianas que refieren riesgo, mayor deterioro e incidencia de las actividades inadaptadas. Ello se traslada a adolescentes que han abandonado la escuela, desocupados y que no realizan otras actividades productivas.

Asimismo constatamos que se realiza escasa o nula intervención social, ya sea pública o privada en el tiempo del ocio. Es decir, existen pocas opciones que no sean un ocio marcadamente consumista, donde si conviven los Adolescentes de trayectoria delictiva con Adolescentes en conflicto con la ley sin haber participado en actividades delictivas. Además el tiempo de ocio se ha estructurado sin la presencia adulta, es decir, sin que exista cohesión, vínculos, espacios de convivencia que a su vez sean educativos, cada vez más los mundos adolescentes y adultos se separan. Coincidimos con los planteamientos de $\mathrm{Ca}$ talano y Hawkins (1996), al señalar que el ocio constituye en estos adolescentes un factor de riesgo, pues incrementa de factor de la conducta desviada, asimismo un ocio estructurado sería un factor de prevención.

\section{Conclusiones y propuestas}

Las necesidades ocio-educativas, requieren respuestas flexibles, directas, en red y transversales a diferentes niveles de lo cotidiano. Las actividades de ocio requieren una adaptabilidad instrumental, a efectos de situar a los beneficiarios de tales acciones en el epicentro de su actuación (Jares, 1999). De nada sirve la estratificación de la dimensión del ocio, pues éste ha de ser inclusivo y de fácil acceso.

El ocio y el tiempo libre en nuestra sociedad ocupan un espacio central en el proceso de socialización, tanto en adolescentes como en adultos; sin duda, una parte del ocio y tiempo libre gira en torno al consumo y por tanto su accesibilidad es un factor de integración, de pertenencia social y en definitiva de inclusión.
Además ha de constituir una oportunidad para la intervención socioeducativa. Coincidimos con Picornell (2004) cuando señala que cualquier acción protectora, además de dirigirse a determinadas situaciones de urgencia, proporcionando ayuda ante necesidades sociales, tiene que posibilitar intervenciones socioeducativas, intersectoriales y coordinadas. La intervención desde el ocio con adolescentes adquiere significado como acción protectora del sistema.

En este sentido proponemos una sociedad en la que el concepto de «educación a lo largo del ciclo vital» conduzca directamente a la noción de sociedad educadora o educativa; una sociedad que multiplique las posibilidades de aprender en cualquier escenario. Los espacios de ocio se han de diseñar en base a tres elementos clave: participación, intercambio y creatividad. Asistimos a un momento histórico en que creamos poco; prácticamente todo lo que se instrumentaliza a los ojos de los adolescentes ya viene dado (juegos de ordenador, entornos virtuales, dispositivos musicales, etc.). El ocio mayoritario que actualmente se consume, deja poco espacio a la creatividad, a la ilusión, a la eventualidad, a la peripecia y al ingenio. Es un ocio pseudo clasificado, que deja poco espacio a la inventiva y a la relación con lo abstracto. El espacio cotidiano llega pues teledirigido por los propios mecanismos que genera el ocio.

Algunos expertos como San Salvador (2000), Garaigordobil (2004), Cuenca (2006), Santos (2010), consideran la infancia como una etapa para el aprendizaje a través del juego. Describen acertadamente el interés semántico del ocio para el desarrollo emocional de los adolescentes. De suma importancia, pues los espacios dedicados fundamentalmente al placer de la diversión han de ser fijados también para la intervención pública, para el acompañamiento social, donde puedan articularse experiencias positivas, integradoras, educativas, solidarias, lúdicas, preventivas e incluso terapéuticas, por tanto ha de ser gratificante tanto para los adolescentes como para la sociedad, que contempla la evolución y el progreso de las actuales adolescencias. En esta línea Pastor (2008) incide en que «las administraciones públicas deben facilitar el acceso y la participación de los ciudadanos no solo como usuarios de servicios y actividades, sino en la 
elaboración, gestión, seguimiento y evaluación de programas» (p. 23). Este protagonismo otorgado al ciudadano permitirá adoptar decisiones consensuadas y vinculantes, provocando una progresiva socialización de la dimensión colectiva del desarrollo local fortaleciendo los recursos humanos y enriqueciendo el debate social. Asimismo, siguiendo a Martínez-Román (2000), debemos seguir un modelo de políticas locales al estilo del local government act del Reino Unido, donde se promociona la incentivación de las estructuras sociales en el ocio común, las estrategias para un ocio independiente a la vez que constructivo, desarrolladas en colaboración entre autoridades locales y entidades del tercer sector.

Abogamos por desarrollar políticas públicas para seducir a los adolescentes hacia las actividades que logren reorientar sus procesos disruptivos. Desde esta óptica, Scandroglio y López (2010) inciden en la importancia de introducir el asociacionismo como fuente de expresión en los jóvenes para transformar la realidad.

Recordando a Parra (2010) diremos que la mejor manera de divertirse, será aprendiendo. Jugando aprenderemos a probar, a intentar, a tolerar más las frustraciones, a prevenir los fracasos, a extraer consecuencias, a sentirnos ganadores, fracasados, a conocer nuestros límites o afianzar nuestros retos. Desarrollar el conocimiento, permite ampliar los valores propios; mucho más si este entrenamiento se realiza desde la niñez y en clave inclusiva que permita una socialización adecuada capaz de impedir segregaciones que eviten procesos de des- viación social. Bajo esta misma idea, Andreu (2003) plantea:

Los responsables de la transmisión de las normas, valores y modelos de comportamiento son los llamados agentes de socialización, que son muchos y que juegan un papel de mayor o menor importancia según la etapa de la vida del sujeto y de su posición en la estructura social (p. 224).

Creemos en un ocio educativo tanto en sus esencias como en sus evidencias, generador de ciudadanía, donde se aúnen la necesidad de vivir el ocio como un derecho individual y social, por tanto como una obligación pública facilitar las iniciativas sociales que lo promuevan y hacer realidad el concepto de ciudades educadoras. De ello se deduce que la ciudad como marco educativo genera muchas posibilidades y la calle, es una escuela de aprendizajes, tanto positivos como de riesgo, pero siempre que exista una planificación del modelo, podremos pues asimilar el espacio abierto a los marcos pedagógicos que favorecen el aprendizaje. La triangulación de todos estos elementos favorece la función social de los individuos, ya que permite sentirse vinculado al espacio en que se muestra representado (Tonucci, 2007). Hay que convertir las ciudades en espacios privilegiados para el aprendizaje, poner la ciudad a los ojos de aquellos que las habitan, diseñando espacios para su inclusión y tiempos para su disfrute. En este sentido, planificar el espacio urbano desde el ocio, ampliar las esferas de lo educativo, desde el mismo intercambio cultural, desde el propio ombligo de la ciudadanía y en aras de un desarrollo plural.

\section{Referencias bibliográficas}

Andreu, J. (2003). Infancia, Socialización familiar y nuevas tecnologías de la comunicación. Portularia, 3, 243-261. Disponible en: http://dialnet.unirioja.es/servlet/articulo? codigo $=808160$ (consultado el 3/11/2012).

Bueno Abad, J. R. y Pérez Cosín, J. V. (1998). Socialización e inadaptación. Una aproximación teórica a la juventud inadaptada. En L.Gómez y F. Jodar (comp.). Sobre la inadaptación (pp. 49-70).Valencia: Burjassot, Gómez Coll.

Caballo, M. B., Caride, J. A. y Meira, P. A. (2011). El tiempo como contexto y pretexto educativo en la sociedad red. Intervención Socioeducativa, 47, 11-24.

Castel, R. (1997). La metamorfosis de la cuestión social. Una crónica del asalariado. Barcelona: Paidos.

Catalano, R. F. y Hawkins, D. J. (1996). The social development model: A theory of antisocial behavior, en Delinquency and crime: Current theories (ed. Hawkins) (pp.149-197). Nueva York: Cambridge University Press.

Cuenca, M. (2004). Pedagogía del Ocio: Modelos y Propuestas. Bilbao: Universidad de Deusto. 
Cuenca, M. (2006) ¿Puede ser la solidaridad una experiencia de ocio? Revista de Proyecto Hombre. $59,9-14$.

Del Barrio, C. (2002). Conflictos entre escolares de educación secundaria y su visión desde el profesorado. Alternativas: Cuadernos de Trabajo Social, 10, 303-318.

Fernández Barrera, J. (2009). Los niños y las niñas: ¿ciudadanos de hoy o de mañana? Alternativas: Cuadernos de Trabajo Social, 16, 111-126.

Funes, J. (2005). El mundo de los adolescentes: propuestas para observar y comprender. Intervención socioeducativa, 29, 78-102.

Garaigordóbil, M. (2004). Programa Juego 10-12 años. Juegos cooperativos y creativos para grupos de niños de 10 a 12 años. Madrid: Pirámide.

Jares, J. (1999). Educación y derechos humanos. Estrategias didácticas y organizativas. Madrid: Editorial Popular.

Lasén, A. (2000). A contratiempo: un estudio de las temporalidades juveniles. Madrid: Centro de Investigaciones Sociológicas-CIS.

Lévesque, M. y Robert, M. (2008). Youth's social networks: influence on their running away behaviours. Portularia, 8, (1). 41-60. http://dialnet.unirioja.es/servlet/articulo?codigo=2797194 (consultado el 16/12/2012).

Martínez Román, M. A. (2000). Cambios recientes en los servicios sociales para menores en Inglaterra. Alternativas: Cuadernos de Trabajo Social, 8, 65-93.

Navarro, J. J. (2009). Las estructuras de bienestar ante los desafíos de la delincuencia juvenil. Servicios Sociales y Política Social, 87, 51-70.

Navarro, J. J. y Puig, M. (2010). El valor de la educación afectiva con niños en situación de vulnerabilidad acogidos en instituciones de protección: un modelo de trabajo social basado en la cotidianidad. Servicios Sociales y Política Social, 90, 65-84.

Parra, M. (2010). La participación como estrategia para la intervención en el ocio educativo de los adolescentes. Arbela: Hezkunza Aldizkaria. 42, 77-82.

Pastor, E. (2008). Mecanismos de participación ciudadana en los Servicios Sociales Municipales. Cuadernos de Trabajo Social 28, 7-25.

Patton, M. Q. (1990). Qualitative Evaluation and Research Methods. Londres: Sage.

Pérez Cosín, J. V. (coord.) (1999). Trabajo social. Orientaciones y prácticas formativas. Valencia: Gules.

Pérez Cosín, J. V. (2010). Políticas para la infancia en el siglo XXI: Desde la asistencia a la defensa un proceso en curso. Servicios Sociales y Política social, 90, 47-64.

Pérez Cosín, J. V. y Uceda, F. X. (2009). Aproximación teórica a los adolescentes en conflicto con la ley: paradigma y modelos. Servicios Sociales y Política Social, 87, 71-92.

Picornell, A. (2004). Modelo definitorio de desamparo infanto-juvenil. Un instrumento para la planificación estratégica. Portularia, 4, 277-286. http://dialnet.unirioja.es/servlet/autor?codigo=282602 (consultado 26/04/2013).

San Salvador, R. (2000). Políticas de ocio: Cultura, turismo, deporte y recreación. Bilbao: Universidad de Deusto.

Santos, M. A. (coord.) (2010). Adolescentes y educación. Madrid. Wolters Kluwer.

Savater, F. (1997). Educar es universalizar. Cuadernos de Alzate: revista vasca de cultura y de las ideas, 16, 7-18.

Scandroglio, B. y López, J. (2010). Investigación-acción-participativa con la agrupación Latin King en Madrid: potencialidades y límites de una estrategia alternativa al control de los grupos juveniles conflictivos. AIBR, Revista de Antropologia Iberoaméricana, 5 (2), 222-255

Tonnuci, F. (2007). Frato, 40 años con ojos de niño. Barcelona: Graó.

Torrente, G. y Rodríguez, A. (2004). Características sociales y familiares vinculadas al desarrollo de la conducta delictiva en pre-adolescentes y adolescentes. Cuadernos de Trabajo Social, 17, 99-115.

Ucar, X. (2001). Cultura y educación social en el marco de la globalización. Pedagogía social: revista interuniversitaria, 6-7, 331-363. 
Uceda, F. X. (2004): De la cosificación a la ternura: reflexiones para un nuevo paradigma en Trabajo Social. Ponencia presentada en III Taller Internacional de Trabajo Social, del 20 al 25 de septiembre de 2004. La Habana (Cuba). Sociedad Cubana de Trabajadores Sociales.

Uceda, F. X. (2012). Adolescentes en conflicto con la ley. Una aproximación comunitaria: trayectorias, escenarios e itinerarios. (Tesis Doctoral inédita). Valencia, Universitat de València.

Vallés, M. S. (1999). Técnicas Cualitativas de Investigación Social. Reflexión Metodológica y Práctica Profesional. Madrid: Síntesis Sociológica. 